\title{
Summary and recommendations of a National Cancer Institute workshop on issues limiting the clinical use of Monte Carlo dose calculation algorithms for megavoltage external beam radiation therapy
}

\author{
Benedick A. Fraass \\ Department of Radiation Oncology, University of Michigan, Ann Arbor, Michigan \\ James Smathers \\ Department of Radiation Oncology, University of California Los Angeles, Los Angeles, California \\ James Deye ${ }^{\mathrm{a})}$ \\ Radiation Research Program, National Cancer Institute, Bethesda, Maryland 20892
}

(Received 21 February 2003; accepted for publication 29 September 2003; published 21

November 2003)

\begin{abstract}
Due to the significant interest in Monte Carlo dose calculations for external beam megavoltage radiation therapy from both the research and commercial communities, a workshop was held in October 2001 to assess the status of this computational method with regard to use for clinical treatment planning. The Radiation Research Program of the National Cancer Institute, in conjunction with the Nuclear Data and Analysis Group at the Oak Ridge National Laboratory, gathered a group of experts in clinical radiation therapy treatment planning and Monte Carlo dose calculations, and examined issues involved in clinical implementation of Monte Carlo dose calculation methods in clinical radiotherapy. The workshop examined the current status of Monte Carlo algorithms, the rationale for using Monte Carlo, algorithmic concerns, clinical issues, and verification methodologies. Based on these discussions, the workshop developed recommendations for future NCI-funded research and development efforts. This paper briefly summarizes the issues presented at the workshop and the recommendations developed by the group. (C) 2003 American Association of Physi-
\end{abstract} cists in Medicine. [DOI: 10.1118/1.1626990]

Key words: Monte Carlo, clinical use, verification, recommendations

\section{INTRODUCTION}

In recent years, there has been widespread interest in the implementation of Monte Carlo (MC) dose calculation algorithms for megavoltage external beam radiation therapy for routine clinical treatment planning. To evaluate the current status of this use of MC, the National Cancer Institute, in association with the Oak Ridge National Laboratory, organized a workshop entitled "Issues Limiting the Clinical Use of Monte Carlo Dose Calculation Algorithms" in Gatlinburg, TN in October 2001. Attendees included experts in clinical radiation oncology, clinical treatment planning, and MC calculation algorithm development and study.

The primary goal of the workshop was the combination of the ideas of all three types of experts to identify issues requiring additional work and support in order to make possible the routine clinical use of MC dose calculations for external beam radiation therapy treatment planning. Specific Monte Carlo codes, their advantages or disadvantages, or commercial MC applications, were not in general discussed. Rather, the goal was to concentrate only on the scientific and clinical issues, particularly those which would benefit from discussion between the clinical and MC experts present at the workshop.

This report gives a brief summary of the workshop discussions, and lists the recommendations for future National Cancer Institute research and development funding which were developed by the workshop. This paper is not a review, a description of Monte Carlo research efforts, or a complete description of all relevant Monte Carlo dose calculations issues or results, but rather a summary of the issues discussed at the workshop.

\section{BRIEF OVERVIEW: MONTE CARLO METHODS FOR RADIOTHERAPY DOSE CALCULATIONS}

Monte Carlo dose calculation algorithms for radiotherapy use the basic physics of particle interactions to simulate the deposition of energy (i.e., dose) in the patient. ${ }^{1-4}$ The MC method propagates individual particles (photons, electrons, etc.) through the treatment machine, and then through the patient, tracking each particle history to determine where energy is deposited along the particle tracks. Individual particle histories are simulated, based on known particle interaction cross sections, particle transport, and energy deposition characteristics. Often, a very large number (millions to billions) of particles must be simulated in order to obtain a reasonably precise estimate of the quantities of interest (e.g., dose), so $\mathrm{MC}$ methods require significant computational resources. Although often quite time consuming, the $\mathrm{MC}$ method is the calculation algorithm that most closely models the actual physics of the energy deposition process, so MC algorithms are expected to be capable of more accuracy than other kinds of calculation algorithms.

Any megavoltage external beam Monte Carlo dose calculation must handle two different parts of the dose calculation 
problem: (1) propagation of the radiation beam through the therapy machine collimation system, and (2) the calculation of dose within the patient, based on the beam which exits from the accelerator head. The latest versions of generalpurpose Monte Carlo codes currently in distribution (EGS4, ${ }^{5}$ EGSNRC, ${ }^{6}$ ETRAN,${ }^{7}$ FLUKA, ${ }^{8}$ GEANT, ${ }^{9}$ ITS, ${ }^{10}$ MCNP,${ }^{11}$ PENELOPE $^{12}$ ) are generally capable of meeting the basic accuracy requirements of radiotherapy, though many have limitations as well. However, they are all generally too slow, taking many hours or days to complete a calculation in the patient (not including the fixed machine-dependent part of the problem). To address this problem, several codes have been developed more specifically for use in radiotherapy, including MCDOSE, ${ }^{13}$ MCPAT, ${ }^{14}$ DPM, ${ }^{15}$ PEREGRINE, ${ }^{16,17}$ VMC,$++{ }^{18}$ XVMC, ${ }^{19} \mathrm{MMC},{ }^{20}$ and Super Monte Carlo. ${ }^{21}$ These codes have reduced the calculation times in the patientdependent part of the problem, in some cases by an order of magnitude or more, sometimes through approximations or compromises including modified electron transport, limited tracking of low probability events, voxel-based transport methods, etc. Careful validation and study of such approximations is of course necessary, and more study of these newer algorithms is required. How to optimize the MC dose calculation method to handle both the machine-dependent and patient-dependent parts of the calculation remains one of the major questions affecting clinical use of this technology.

The rationale for using $\mathrm{MC}$ dose-calculation algorithms for clinical planning include improved accuracy for (1) inhomogeneities, (particularly for lung and bony anatomy), (2) tissue interfaces (lung interfaces, the airway, sinuses), and (3) very small fields (including those used in IMRT treatments) that also exhibit lateral electron disequilibrium effects. Other expected advantages of Monte Carlo include the following:

(a) The improved accuracy of MC techniques should apply to all anatomic geometries, all modalities and all shaping devices.

(b) MC should lead to increased confidence in the accuracy of dose distributions.

(c) MC should eliminate the need to develop new and more complex dose calculation models.

(d) MC should eliminate the laborious trial and error parametrization which is necessary with most current model-based calculation algorithms.

(e) $\mathrm{MC}$ algorithms may reduce the amount of measured dose distribution data required for beam and dose calculation characterization during new machine commissioning.

(f) MC will allow direct calculation of monitor units, hopefully leading to a reduction in the probability of human mistakes.

(g) MC should allow accurate estimation of quantities difficult or impossible to measure (for instance, dose perturbations from small inhomogeneities in vivo).

\section{ISSUES FOR MONTE CARLO ALGORITHMS}

Most general purpose MC codes, when applied to the megavoltage radiotherapy problem, are too slow and cumbersome. To gain speed, most MC algorithms designed for use in planning divide the calculation into three different parts, including (1) the fixed beam in the head of the machine, (2) propagation through the field-dependent collimation and beam modifiers, and (3) the transport through the patient. The first session of the workshop reviewed the status of some current $\mathrm{MC}$ algorithms with respect to the following basic components of MC algorithms.

\section{A. Photon transport and cross sections}

The first part of a Monte Carlo dose calculation simulates the radiation transport through the head of the treatment machine (x-ray target, flattening filter, monitor unit chamber, primary collimator) and converts the electron beam into a relatively flat, wide beam of $\mathrm{x}$-ray photons. This is a time intensive calculation due to the transport of electrons through the high atomic number materials used in the head, and significant absorption of photons by the primary collimator and flattening filter means that more particles must be simulated in order to achieve a statistically meaningful result, contributing to the relative inefficiency of the treatment head simulation. However, the treatment head simulation is typically only conducted once, for each beam energy, since it is typically assumed that the linear accelerator remains stable with respect to beam energy. ${ }^{16,22-24}$

The field-specific parts of the calculation involve transport through beam modifiers that significantly attenuate parts of the photon field. Unfortunately, this also implies a large number of photon interactions and consequently a large amount of computer time. Approximations for this aspect of the calculation have been attempted, ${ }^{25}$ but are not without difficulties. ${ }^{17}$

Transport within the patient is also time consuming. The patient is typically described by a CT grid, that has a fine resolution relative to the photon mean free path; so each photon experiences many boundary crossings, each of which increases the computational effort. Various methods have been developed to address this problem. ${ }^{17,26-29}$

The three main photon interaction processes that occur are photoionization, Compton scattering, and the production of electron-positron pairs. A detailed review of the state of the various cross-section data sets was presented. In general it was felt that the quality of these data were not the limiting factor in current MC implementations, however, sensitivity studies are needed to further test that hypothesis. Differences in bremsstrahlung data resulting in differences in absolute photon output have been reported in machine modeling studies. Preliminary studies of the sensitivity of calculations to a free versus bound Compton scattering model were also discussed, and more formal benchmark trials that test the underlying data and their effect on accuracy and efficiency were suggested. 


\section{B. Electron transport}

Electron transport is a crucial component of any external beam MC calculation method, since photons set electrons into motion and the electrons then deposit energy in tissue. The transport of electrons is an important aspect of the calculation that involves very large numbers of smaller interactions due to the long-range Coulomb force. Collision-bycollision transport of electrons is clearly untenable for practical applications, so most radiotherapy MC codes utilize the condensed history method. ${ }^{7,30}$ In this method, the electron's path is divided into sub-steps, and the energy loss and angular deflection at each step are averaged over many individual collisions. Electron transport algorithms are often categorized as Class I or II models based on how the energy of the primary electron is related to the energy lost in individual interactions (MCNP, ${ }^{11} \mathrm{ETRAN}^{7} \mathrm{EGS4}^{5,6}$ ). Since the electron transport may be crucial to some radiotherapy calculations, further investigation and validation of electron transport algorithms is needed.

\section{Photoneutron transport and cross sections}

Many therapy accelerators use energies high enough to involve photon-neutron interactions. Fairly comprehensive photoneutron cross sections are available for major body elements such as $\mathrm{C}, \mathrm{N}, \mathrm{O}$, and $\mathrm{Ca}$ within the photon therapy energy range of interest. Data on specific particle production channels are often missing, though there is an excellent summary of current photonuclear measured data compiled by the International Atomic Energy Agency. ${ }^{31}$ The photonuclear component contributes very little to energy deposition in the human body, and since one can decrease this component further by lowering the energy of the beam, it is reasonable to question the need for further detailed investigations of this process with MC. This effect is likely very small in tissue and most neutrons will escape the patient volume. However, the activation of metallic implants has yet to be investigated in detail, and requires more effort.

Neutron generation in machine elements and in therapy room shielding is a problem recognized in the clinical setting. Photoneutron production is a measurable quantity in the treatment room, and there are suggestions that it might limit the time the therapist can spend near the facility. Measurements in clinical settings are now being undertaken by some researchers, although these quantities are difficult to measure due to the mixed radiation fields and the large uncertainties associated with the data. ${ }^{32-34}$

\section{Patient tissue identification issues}

A number of techniques have been used to correlate CT number with a particular elemental tissue compound and corresponding mass density, since these are important pieces of information required by Monte Carlo methods. The photon mass attenuation coefficient for various tissue compounds, as defined by ICRU $44,{ }^{35}$ shows that all tissue compounds are basically identical for the radiotherapy energy range of interest $(0.1-10 \mathrm{MeV})$, since Compton scattering is the dominant photon interaction process, and it is only dependent on the electron density of the materials. Considering energy deposition, only adipose tissue and cortical bone show large differences in total electron stopping power relative to water, mainly due to the lower atomic number of fat and the higher atomic number of cortical bone. To improve the consistency of the various MC algorithms, however, it would be useful to utilize a consistent method for conversion of CT numbers-totissue identification (the analysis of Schneider et al. could serve as a good starting point ${ }^{36}$ ).

\section{E. Variance reduction techniques}

One of the unusual aspects of Monte Carlo dose calculations is that evaluation of the dose distributions has to accommodate the fact that $\mathrm{MC}$ uses a stochastic technique, so there is a variance associated with the dose result at each point. Since increasing the precision (and decreasing the variance) can require significant additional calculation time, variance reduction is an important topic of current research. Improved variance reduction techniques may help decrease calculation time if they can be applied with appropriate care. In addition, variance reduction is a good example of an issue that clinical medical physicists and physicians must understand in order for MC calculations to be accepted (and properly used) in the clinic. Further education in this area is clearly warranted, particularly with the goal of describing the differences between statistical variance reduction, systematic errors, and de-noising, since these issues are sometimes confused.

\section{F. Machine description and/or source modeling}

Describing the radiation beam which comes from the accelerator; and commissioning the dose calculation results, which are obtained from the Monte Carlo dose calculation algorithm (i.e., assuring that the calculation results agree with measured data for the particular machine being modeled), are very important aspects of any dose calculation model, and this is still true for Monte Carlo dose calculation algorithms. Accurate simulations of radiation transport in the treatment machine (and then the patient) rely on knowing the characteristics of the radiation beam that comes through the machine's head, and in principle includes knowing the position, velocity, direction of motion, charge, and energy of each particle which contributes to the output of the machine. Since the clinical physicist cannot measure these physical phenomena directly, this can become a daunting task at the level of the treatment planning facility.

For Monte Carlo dose calculations in the patient, the most common way to obtain this description of the beam is to perform a Monte Carlo simulation of the beam, typically starting with the electron beam impinging on the photon target of the machine, and propagating all the particles created throughout the machine. Much of the work on this aspect of the process has made use of the BEAM code, developed by the Omega/Beam project collaboration. ${ }^{22}$ Since the machine head simulations require a great deal of calculation, the simulation is typically performed just once for each machine 
energy, scoring or storing the results of the particle simulations in the head above the collimator jaws or any other moving parts of the collimation system. The MC calculation for each radiation field then begins from this starting point.

Several different approaches with regard to the head simulation data are used for patient MC calculations. The "phase space" method records the location, direction, and energy of each particle in the head simulation at some scoring plane located above all the movable parts of the collimation system. This phase space description is then the input into the MC calculation used for the field- and patientspecific dose calculation. The storage requirement for the phase-space files generated by this method, containing millions of photons and electrons, are very large (tens of gigabytes ${ }^{37}$ ). Before this kind of method can be used for clinical calculations, the MC simulation of each beam in the radiotherapy clinic must be performed, a calculation that is time consuming and dependent on detailed knowledge of the accelerator head geometry and materials. The correctness of the phase space description must be confirmed by comprehensive commissioning checks (comparisons of calculation results and measurements). Correctly performing these calculations and checks requires a high level of sophistication and experience with Monte Carlo calculations.

Rather than directly using the phase space data, it is also possible to create a model of the phase space distribution that recreates the phase space without saving the actual distribution of particles. Making use of a source model can save considerably on disk space. ${ }^{24,37-41}$ Using such a source model, the phase space can be reconstructed and fed into the dose calculation code one particle at a time, eliminating the need to store phase-space data. This approach has been used for both photon beams ${ }^{42,43}$ and electron beams. ${ }^{44}$ Another method which has been used is based more directly on measured input data, analogous to the way many current nonMonte Carlo algorithms use measurement-determined parameters.

A number of issues remain to be solved for source modeling and beam commissioning. How sensitive the different $\mathrm{MC}$ approaches are to machine-specific variations (in accelerator tuning, mechanical tolerances, materials, etc.) is not known (see Sec. V D). Differences that result from use of the phase space description directly versus that of a source model have not been completely described. Comprehensive verification of any source model should be performed, and the verification criteria that will be used should be further investigated. Currently, source model accuracy is determined by the agreement between measured dose distributions in different phantoms and the calculated doses using the reconstructed beam data, but other end points (e.g., particle fluence or energy and angular distribution) may be necessary. Development of effective and efficient beam commissioning methods and software will facilitate the widespread clinical application of MC treatment planning.

\section{MONTE CARLO DOSE CALCULATIONS IN THE CLINIC}

\section{A. How will improved accuracy be used by the clinic?}

In the treatment of cancer with radiation, the radiation oncologist must select a treatment regimen that will have a high probability of curing or controlling the disease while not inflicting undue and/or unexpected complications on the patient. Determining the best way to perform the treatment is made difficult by many factors including the basic doseresponse relationships for both tumor and normal tissue. In general, published clinical and experimental results show tumor control or normal tissue effect response as a very steep function of radiation dose. For example, a small change in the dose delivered $( \pm 5 \%)$ can result in a dramatic change in the local response of the tissue $( \pm 20 \%){ }^{45,46}$ Moreover, the prescribed curative doses are comparable to and often exceed normal tissue tolerance doses. Thus, for optimal treatment, the radiation dose must be planned and delivered with a high degree of accuracy. While it is difficult to assess how accurate the overall process should be, the ICRU ${ }^{47}$ recommends that dose be delivered with an error less than 5\%. Thus, each step (machine calibration, patient positioning, dose calculation, etc.) needs to be performed to an accuracy much better than 5\%. For the dose calculation step, the necessary accuracy is believed to be on the order of $2 \%-3 \%$. $^{48}$

The gain to each individual patient from the use of more accurate dose distributions is not measured simply as the difference in dose calculated by current and improved methods, as the difference will be relatively small in most cases. Rather, the gain will be reflected by either increasing probability of tumor control and/or decreasing rates of complications, both of which are results that may become more probable if more precise knowledge of the dose distributions is available. More accurate dose distributions should allow physicians to make better clinical planning decisions, once the more accurate doses are correlated with clinical knowledge. Most treatment plans today include margins for safety that make complications rare, so improved compliance with physician specified tolerance limits due to $\mathrm{MC}$ calculations may be hard to demonstrate. On the other hand, tumor recurrences are common events, and the opportunity provided by more accurate dose calculations to raise tumor dose without violating specified constraints in normal tissues can be used to advantage in clinical practice. Likewise, consistently more accurate dose calculations will enhance clinical research by potentially "sharpening" the dose response curves and thus increasing our knowledge about the radio-response of diseased and normal tissues.

As optimization techniques in radiotherapy delivery improve, one will be able to treat the tumor to the highest dose possible while keeping the dose to critical tissues at their tolerance limits. When plans are optimized in this way, tumor dose is found to be very sensitive to the exact specification of the dose constraints: variations as small as a few percent in the specification of a volume fraction that can exceed a critical dose can cause a much larger change in the minimum tumor dose which can be delivered, ${ }^{49}$ and this can 
force a change in the plan technique which should be used. A similar effect on minimum tumor dose can be seen when the dose homogeneity limit for the target is varied. ${ }^{50}$ The small variations in the dose specifications that induce these large changes in minimum tumor dose fall well within the range of uncertainty in current estimates of the dose distributions. The tolerance limits themselves can become better refined by allowing better correlations between dose and adverse events in large scale clinical trials, a research topic which would benefit from improved support.

\section{B. Is electron Monte Carlo useful and/or necessary in the clinic?}

Electron beam treatments typically represent about $10 \%-$ $15 \%$ of the daily workload in clinical practices, and are used prominently as boosts for relatively superficial targets. For this kind of standard use, the electron beam to be used is chosen clinically, and the dose distributions are generated primarily for treatment documentation. There has also been some sophisticated clinical use of electron beams which might benefit from improved dose calculation accuracy (as compared to the standard clinical use described earlier), but the limited accuracy of current non-Monte Carlo algorithms, even three-dimensional pencil beam algorithms, has probably impeded the integration of sophisticated electron treatment planning into routine clinical practice. Significant differences between pencil beam dose distributions and $\mathrm{MC}$ calculations have been demonstrated, particularly in regions near air cavities and/or bones, with oblique incidence, small irregular fields, and with extended SSDs. ${ }^{51}$ As electron beam Monte Carlo algorithms begin to appear in commercial planning systems, it will be important to explore the treatment planning situations in which accurate dose calculations will be critical-including small fields, electron IMRT implementations, clinical sites with bone or low density tissues, and complex delivery techniques like electron arc therapy or the use of MLCs for electron beams. Further research in Monte Carlo based electron dose calculations will be important for these clinical uses.

\section{Monte Carlo and IMRT}

Monte Carlo calculation algorithms may find significant importance for Intensity Modulated Radiation Therapy (IMRT) planning, and a number of issues in this area need to be considered. ${ }^{52,53}$

(1) IMRT is typically delivered with either a dynamic or static sequence of small fields, so techniques need to be developed for modeling the intricate MLC designs in a "sufficiently" accurate way, while at the same time allowing the calculations to be performed rapidly. IMRT is one of several techniques which involve relatively small field irradiation techniques (stereotactic radiosurgery is another), and this is an area of radiation oncology in which Monte Carlo dose calculations may make a significant difference, ${ }^{54}$ since lateral disequilibrium effects can become large, and the differences can be further exacerbated by the presence of low density tissue heterogeneities.
(2) Depending on the type of inverse planning or optimization approach that is used, IMRT dose distribution calculations need to be repeated hundreds, or even thousands of times during the optimization process. Schemes must be developed to accomplish this expeditiously without compromising the accuracy afforded by MC techniques.

(3) It is well known that for situations like single beams passing through slab-like low density inhomogeneities, conventional calculation methods can lead to very large errors near the beam boundaries or even in the middle of a small beam, especially for high energies. Superposition/ convolution algorithms improve on this result, but are very much slower, and do not remove all the errors. MC can give improved accuracy for these potentially crucial aspects of the IMRT planning process, especially if increased calculation time does not overshadow the improved accuracy of the calculations.

(4) IMRT deliveries depend on complex MLC shapes for which the calculation of head scatter effects is exceedingly difficult, yet increasingly important. MC should be able to provide accurate calculation of the head scatter for the very complex DMLC (dynamic MLC: movement of the MLC with the beam on) or SMLC (segmental MLC: multiple fixed MLC segments) patterns that are often used to deliver IMRT.

\section{Operational issues}

A number of additional operational issues that can complicate the clinical acceptance and use of MC dose calculations were described.

(1) User confidence: As with any other dose calculation method, errors in software coding, input data, or other implementation problems can potentially lead to computational artifacts in the dose distribution results. It is possible that an artifact may be large enough to cause clinically important errors, yet subtle enough to not be detected by experienced plan evaluators, especially those used to the results from more simplistic and approximate calculation algorithms. Due to the expectation that $\mathrm{MC}$ will be very accurate, possible artifacts may be accepted as real because the perception is that "Monte Carlo is more accurate."

(2) Calculation speed: MC calculation algorithms are still slower than desirable for routine clinical use, though the situation is continuing to improve. Workshop attendees stated that the time necessary for dose calculations should not exceed 10 min for a typical $4-6$ beam plan, as a goal. For benchmarking or retrospective studies, longer times (a couple of hours) are reasonable, though faster is better. It was the sense of the group that current MC algorithm/hardware combinations are becoming fast enough to address this concern, but that further work is required to document that the expected accuracy of the MC calculations is not lost due to speed-related compromises in the faster algorithms.

(3) Accuracy: MC is expected to be significantly more accurate than standard methods, and excellent agreement with measurements has been shown by numerous researchers. However, achieving this high level of accuracy has not been shown to be routinely achievable without significant 
amounts of beam-specific tweaking of the MC algorithm and/or parameters.

(4) Calibration: Ideally (with a perfect description of the machine), it should be possible to calibrate MC output for each beam quality via a single reference set of conditions (e.g., dose/unit fluence at reference depth/SSD for one field size). However, at present, empirical adjustment is still needed.

(5) Statistical fluctuations: Handling statistical fluctuations in the dose distribution present considerations that are not present for current deterministic calculation algorithms. The size of these effects may be decreased by the proper application of variance reduction techniques, however, new techniques to handle these statistical methods may be useful.

(6) Prescription paradigm: It will likely be necessary to educate clinicians to not prescribe or evaluate biological effects based on dose at any one specific voxel, since the MC dose to any voxel is only known with a given statistical uncertainty. Even if we choose to allow a statistical uncertainty of $1 \%$ in the dose calculation results, that does not mean that we want the entire dose distribution renormalized by $\pm 1 \%$.

(7) Dose to what?: Considerations of dose to water versus dose to tissue become relevant with $\mathrm{MC}$ since the medium of dose deposition is required for the calculation. One can certainly report the doses either way, but at the current time, there is no significant advantage of one over the other. Clinically, the differences may be relatively unimportant except perhaps at high energies in bone.

(8) Dose resolution: What resolution is appropriate for the dose distribution, particularly near tissue interfaces, has not been determined. This issue is quite important because the voxel sizes used have a significant effect on calculation time and interface dose accuracy.

(9) Transition zones: Contaminant electrons figure into the dose in the buildup region, always a problematic region for dose calculations. Current MC methods have not really solved this issue, ${ }^{55}$ probably due to incomplete solution of the machine head simulation and/or other limitations to the phase space of the particles incident on the patient. Likewise, high- $\mathrm{Z}$ implanted materials continue to be a calculational problem, since $\mathrm{CT}$ artifacts must be eliminated for any algorithm, including MC, to achieve accurate results. Currently, manual editing of CT-derived electron densities is the only method for handling these problems, and this process is time-consuming and subjective method. Further work is clearly warranted.

\section{E. Clinical sites for which Monte Carlo may be useful}

The clinical treatments that will benefit most from improved dose calculation accuracy are those for which the improved accuracy makes possible better informed decisions about clinical plan optimization. These improved decisions will include how to deliver more dose to the tumor without compromising safety and better knowledge of dosimetric limits for normal tissues. Clinical sites which involve tissue inhomogeneities or interfaces between regions of different density are likely to benefit, as are sites treated with small fields, sharply varying intensity distributions, complex sets of beam modifiers, or other complex treatment techniques which lead to lateral electron disequilibrium or other effects which are typically only handled correctly by MC algorithms. Treatments in the head/neck, and thorax (lung, breast/ chestwall, etc.) are thus obvious candidates due to the significant inhomogeneities involved, but other treatment sites may also be important. Even air filling the rectum can cause unexpected perturbations to the dose distribution in treatment of prostate cancer. The shaping of high dose regions around the clivus in nasopharynx tumors may, in principle, allow improved coverage of the target; but the advantage to the patient will be lost if the high-dose region drifts into the adjacent brain stem because of inadequate accounting for the effect of surrounding air cavities. Similarly, treatment of small lung tumors in patients with limited pulmonary reserve may be greatly advanced by reducing the field margins using techniques of intensity modulation. Perhaps it will be possible to reduce the field width by modifying the intensity profile to compensate for the underdosing at the edges of the target that would otherwise occur. The required modulation is difficult to plan correctly with current dose calculation engines, and implementation would greatly benefit from the accuracy that $\mathrm{MC}$ methods could provide.

Many of these issues become more important for intensity modulated radiotherapy (IMRT) treatments, since IMRT treatments involve complex intensity distributions delivered by static or dynamic methods which often involve very small component beams which will be strongly influenced by lateral electron disequilibrium effects. The tightly shaped and complex dose distributions created by IMRT demand a high degree of accuracy, and non-MC methods may not be capable of the desired accuracy. It is anticipated that MC methods, by correctly taking into account the entire patient and delivery system, may be able to significantly decrease the differences between planned and actually delivered doses, thereby allowing development and delivery of improved treatment regimens.

Much of our current knowledge of dosimetric limits for normal tissues is based on simplistic dose calculations. In order to maintain safety, gain clinical acceptance of the MC method, and determine the realistic implications of use of more accurate dose calculations, it will be extremely valuable to sponsor studies which recalculate the dose distributions of retrospective series of patients using MC methods, and then relate the new and more accurate dose distributions to clinically observed rates of complications and/or tumor control. With these kinds of studies, we can make use of already obtained clinical data to help us understand specifically where MC dose calculation methods will contribute most to the improvement of patient outcomes.

\section{MONTE CARLO DOSE CALCULATION VERIFICATION}

Before any new technology is implemented for clinical use, the safety and appropriateness of the technology should 
be confirmed. For MC dose calculations, this reduces to two highly related tasks: validation and verification. Here we will take validation to be the process by which we confirm that the MC calculations work as designed (and that appropriate databases and cross sections are used), and verification to be the checks that the MC calculations give good predictions of the actual dose distribution that is (or would be) measured for each situation. Given the complexity of MC codes, validation is often assumed by the user based upon the "pedigree" of the code or it is folded into the verification process which compares the calculated results to those obtained by direct measurement.

\section{A. Scope}

The design of a verification program for MC dose calculation methods is complicated by the fact that MC methods are used for two distinct purposes: routine use for clinical treatment planning, and as a benchmark calculation, often for situations in which measurements are difficult or impossible to perform accurately. The scope of verification testing necessary for these two purposes is quite different.

The testing required for clinical use of any dose calculation algorithm has been described in many publications, including the report of AAPM Task Group 53. ${ }^{56}$ The main difference for a MC-based algorithm is that, if the algorithm claims to be more accurate at interfaces between tissues, or for small fields with lateral electron disequilibrium effects (for example), then the commissioning tests should document that the claim is appropriate. All clinical commissioning is aimed at demonstrating those areas in which the algorithm is accurate, and documenting those situations in which there is less accuracy than desired (or claimed).

The scope of verification testing required for a MC code to be used for benchmark calculations, or for situations which are too difficult to measure directly, is much larger. The MC calculations are in this case expected to give the correct answer, and the verification testing must convince the users that the MC calculations are in fact right. To do this, the MC modeling of the physical processes involved must be quite complete, and the capability of the algorithm to "extrapolate" past situations in which measurements can be made must be confirmed by a combination of measurement, theoretical analysis, and careful algorithmic testing. How to do this is not straightforward, since our ability to make accurate dosimetric measurements in these complex situations gets reduced as the situations get more complex-so the interpretation of differences between MC calculations and measurements gets harder to interpret as situations become more complex. Further work on more sophisticated measurement techniques or other methods that can help confirm the accuracy of MC calculation results is needed.

\section{B. Verification experiment design}

Many different strategies have been used for designing verification experiments for dose calculation algorithms. The kinds of problems for which a MC algorithm is most applicable are quite different than the standard situations ad- dressed by model-based algorithms, and the testing requirements are thus different. The design of MC verification experiments should depend on carefully prescribed experimental goals, and should use measurement techniques that are confirmed to be accurate in the situations involved. Issues to be resolved include the following:

(1) Decision on the scope of the verification that is being pursued.

(2) What minimum set of measurements is needed to benchmark a particular algorithm?

(3) What measurement limitations are deemed acceptable in order to allow extraction of meaningful benchmark results?

(4) How does one validate the accuracy of the MC code(s), particle transport in the patient, and the derived source phase space independently?

(5) Can earlier benchmark data be used (for example, the benchmark inhomogeneity measurements by Rice $^{57}$ as recommended by AAPM TG $53^{56}$ ), or are those situations and data too limited to be adequate for the MC testing?

(6) Should the testing concentrate on situations that stress the algorithms (for example, perhaps the accuracy of transport is best examined at small field sizes, which have minimal source model requirements, and in heterogeneous media, to emphasize the loss of lateral electronic equilibrium)?

(7) How much effort should be expended to study the influence of physics transport parameters (such as the low energy electron cutoff) on the final dose calculation in heterogeneous media?

Given that MC dose calculations are far more sophisticated than other calculation algorithms, the level of testing needs to be more complete. A general consensus among workshop attendees supported the organization of a working group to investigate MC algorithm testing, and particularly the development of one or more benchmark data sets that could be used to qualify various MC approaches or algorithms. Since different MC approaches use different approximations or limitations, benchmark verification data sets should include tests and input from different investigators with different strategies. Also, agreement on how to measure the data is essential, and the consensus of a working group involved in the measurements will be crucial to the data set(s) being considered as a true benchmark result.

\section{Verification results for MC algorithms}

A number of presentations on the current state of MC algorithm verification results were presented. Particularly within the last two or three years, there have been a number of publications comparing MC calculations and measured data for a number of MC codes. ${ }^{13,17-19,39,40,51,58-68}$ However, it was clear from the workshop presentations, as well as the literature, that full sets of verification experiments have not been completed for any of these codes, and that much work remains to be done in this area. Decisions about issues to be 
pursued, methodology, and specific experiments that should be performed would be an excellent task for a working group aimed at establishing the basic verification requirements for $\mathrm{MC}$ algorithms and their clinical use.

\section{How important are machine-specific variations?}

For most calculation algorithms, parameters of the calculation model are set by the local physicists to "fit" the calculation results to the measured data for each beam of each accelerator. Whether this mode of operation was appropriate for MC algorithms led to much discussion:

(1) A number of beam parameters for each accelerator are "tweaked" during installation: does this mean similar MC parameters should also be "tweaked" locally?

(2) How might these algorithm parameter adjustments affect the accurate modeling of the beam using MC?

(3) Should adjustments be used to compensate for manufacturing variations, such as slight misalignment of the flattening filter?

(4) Is it inappropriate to alter actual physical parameters, such as the assumed shape or position of the flattening filter, in order to reach agreement between measured data and calculations?

(5) Should individual clinics modify MC parameters to force agreement between $\mathrm{MC}$ calculations and measurements in areas where measurements have significant error bars?

(6) Are the MC calculation predictions more accurate than the measurement system in some centers, or in particular dosimetric regions (e.g., the build-up region)?

(7) How can accelerator manufacturers be convinced to distribute accurate detailed geometrical and materials information on their equipment, so that each individual clinic is not forced to model each accelerator system independently?

(8) Can a quick and easy-to-use MC modeling tool be developed to help users with the difficult machine modeling task?

(9) Should manufacturers provide a standardized set of output data, including phase-space files, that could be used for quality control of individual modeling attempts?

(10) How much of the accuracy of the MC calculations is dependent on the specifics of the individual machine, machine modeling, and local beam measurements?

\section{E. Clinical verification by user}

Most calculation algorithms used in clinical treatment planning are commissioned by the local user, to make sure that the calculations agree adequately with the locally measured data, and to document disagreements or limitations of the algorithm. In the case of MC calculations, it is difficult to determine how much of this clinical verification testing should be performed locally. All of the above-discussed issues (in Sec. VD) also apply here to clinical verification checks. As concluded above, more experience, sensitivity analysis, and independent verification checking is necessary before there is enough experience to determine a recommen- dation about how much local verification checking should occur. This would be a good topic of investigation for a MC working group.

\section{F. Clinical trials groups and MC}

Radiation treatment planning and dose delivery with the advanced technologies such as 3DCRT and IMRT is not as straightforward as with conventional radiotherapy techniques. More precise definition of tumor and normal tissue is necessary as is the need to evaluate all aspects of the treatment plan in three dimensions instead of the more familiar two dimensions. Multi-institutional clinical trials utilizing these advanced technologies require a rigorous QA program. ${ }^{69,70}$

It is possible that use of $\mathrm{MC}$ algorithms may become practical for credentialing and QA review over the next 3 to 5 years for: (1) 3D CRT with $\mathrm{x}$ rays, (2) serial tomotherapy IMRT with binary MLC, (3) cone-beam IMRT with full-field MLCs, and (4) stereotactic radiotherapy using fields collimated by cones and microMLC. MC could be used to validate dose distributions generated by specific vendor planning and dose delivery systems, including IMRT systems. There is also potential to use MC codes for recalculation of dose distributions stored in the Image-guided Therapy Center's (ITC) database, e.g., the RTOG 93-11 lung data which involves significant tissue heterogeneity. However, before MC simulation is practical for use in clinical trials QA, a great deal of research and development remains; particularly with regard to careful and detailed verification testing of the MC codes to be used.

\section{SUMMARY OF RECOMMENDATIONS}

Recommendation 1: Clinical verification of $M C$ algorithms. Many of the issues believed to be the highest priority for additional investigations and research could be very effectively handled by a collaborative working group which includes experts in treatment planning and dose calculation algorithms, MC algorithms, and measurements. The group should be charged to evaluate Monte Carlo algorithm issues, and to determine the best way to overcome the limitations that have been discussed in this report. Specific goals of this group should include the following:

(1) Development of standardized Monte Carlo calculation algorithm benchmark data sets, and an algorithm verification procedure based on use of the benchmark data.

(2) Algorithm comparisons using benchmark test cases (experimental measurements in homogeneous and heterogeneous phantoms), in order to determine which approaches and approximations are best in terms of clinical implementation issues. Specific approaches to validation issues, variance reduction, de-noising, electron transport models, and other issues should be studied, compared, and evaluated.

(3) Development of a process that would promote distribution of accurate MC machine modeling data from manufacturers. 
(4) Determination of methods to validate phase-space models or simulations of accelerator head geometry.

(5) Development of methods for clinical evaluation and use of dose distributions with variable statistical uncertainty.

(6) Study of the sensitivity of the Monte Carlo dose calculations to uncertainties in machine design, machine tuning, and changes known to occur in accelerator components over time.

Recommendation 2: Study of the potential clinical impact of $M C$. Once the improved accuracy of MC algorithms is documented, it is crucial to determine how physicians should learn how to use this more accurate information. Retrospective dose assessments of already existing clinical complications and local control data, using doses predicted with improved accuracy MC algorithms, may give an early indication of the clinical benefit of MC calculations, and may also help physicians determine how to use the new MC doses. Data from retrospective analyses should eventually show us how to make use of this information in a prospective way. This could also effectively be performed using a collaborative working group

Recommendation 3: Further MC research areas. A number of further developments of the Monte Carlo algorithms should be investigated:

(1) Methods to help to simultaneously confirm accelerator head modeling, the phase space of particles exiting the machine, and transport through the patient.

(2) Further study, extension, and verification of the machine model, including phase-space and source modeling methods to all relevant situations for radiotherapy dose calculations.

(3) Additional study of variance reduction techniques, improved computational techniques including parallel processing, improved software techniques, and de-noising are all warranted. What is the sensitivity of the results for each of these techniques, and what compromises do they introduce?

(4) Improved electron transport, particularly attempting to speed the implementation of these calculations.

(5) MC algorithm sensitivity analysis to determine how sensitive dose results are to various data and features of the MC method. How accurately does each parameter need to be determined, and which kinds of uncertainty can lead to significant potential errors in the predicted dose distributions?

(6) Improved implementation of IMRT delivery techniques into the MC algorithms, plus verification that the calculational results accurately predict the actual dose distributions.

(7) Integration of $\mathrm{MC}$ calculation algorithms into the inverse planning/optimization process for IMRT in a timeeffective way.

Recommendation 4: Continuing education. Education of physicists and physicians on the methods and clinical implications of Monte Carlo algorithms and their implementation is crucial. This should include symposia and training as well as guidance materials from the vendors and societies. Without these efforts, there is the risk of accepting the potential superior accuracy of MC as fact; while, in reality, it may be worse than existing computations in clinics which do not properly implement and understand the method.

Recommendation 5: Brachytherapy, internal sources, intravascular brachytherapy. The discussion of the uses of brachytherapy, internal sources, and intravascular brachytherapy related dose calculations using Monte Carlo was beyond the scope of the present workshop. It was recommended by the current workshop that additional workshops should be directed toward such uses, in order to review the field and determine which issues would benefit from further attention by the National Cancer Institute.

\section{ACKNOWLEDGMENTS}

Sponsorship of the workshop was provided by the National Cancer Institute, National Institutes of Health. In addition, the authors would like to thank the staff at the Oak Ridge National Laboratory for their aid with the logistics of this workshop as well as their scientific input during the actual presentations and discussions. We are especially appreciative of the efforts of Bernadette L. Kirk, Group Leader, Nuclear Data and Information Analysis, who gave generously of her time and knowledge toward accomplishing the goals of the workshop.

\section{APPENDIX: PARTICIPANTS}

Organizing Committee (alphabetical): James A. Deye, Ph.D. (NCI), Benedick A. Fraass, Ph.D. (University of Michigan), Bernadette L. Kirk (Oak Ridge National Laboratory), James B. Smathers, Ph.D. (University of California, Los Angeles).

Other Participants (alphabetical): Rosemary Altemus, M.D. (NCI), John A. Antolak, Ph.D. (UT MD Anderson Cancer Ctr.), Paul M. Bergstrom, Ph.D. (National Inst. of Standards \& Tech.), Alex F. Bielajew, Ph.D. (Univ. of Michigan), Indrin J. Chetty, Ph.D. (Univ. of Michigan), Bruce Curran, ME (NOMOS Corporation), John J. DeMarco, Ph.D. (University of California, Los Angeles), Iwan Kawrakow, Ph.D. (National Research Council of Canada), Mark Langer, M.D. (Indiana University), Dennis D. Leavitt, Ph.D. (Univ. of Utah School of Medicine), C. M. Charlie Ma, Ph.D. (Fox Chase Cancer Center), Radhe Mohan, Ph.D. (UT MD Anderson Cancer Center), James A. Purdy, Ph.D. (Washington Univ. School of Medicine), Julian G. Rosenman, M.D. (University of North Carolina), Jeffrey V. Siebers, Ph.D. (Virginia Commonwealth University), Timothy D. Solberg, Ph.D. (University of California, Los Angeles), Michael G. Stabin, Ph.D. (Vanderbilt University), Rosemary S. Walling, Ph.D. (Lawrence Livermore National Laboratory), Laurie Waters, Ph.D. (Los Alamos National Laboratory), Ellen D. Yorke, Ph.D. (Memorial Sloan-Kettering Cancer Center). 


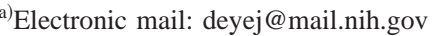

${ }^{1}$ R. Mohan, C. Chui, and L. Lidofsky, "Energy and angular distributions of photons from medical linear accelerators," Med. Phys. 12, 592-597 (1985)

${ }^{2}$ D. W. O. Rogers and A. F. Bielajew, "Monte Carlo Techniques of electron and photon transport of radiation dosimetry," Chapter 4 in The Dosimetry of Ionizing Radiation, Vol. III, edited by K. R. Kase, B. E. Bjarngard, and F. H. Attiz (Academic, 1985).

${ }^{3}$ P. Andreo, "Monte Carlo techniques in medical radiation physics," Phys. Med. Biol. 26, 861-920 (1991).

${ }^{4}$ P. L. Petti, M. S. Goodman, T. A. Gabriel, and R. Mohan, "Investigation of buildup dose from electron contamination of clinical photon beams," Med. Phys. 10, 18-25 (1983).

${ }^{5}$ W. R. Nelson, H. Hirayama, and D. W. O. Rogers, The EGS4 code system-Report SLAC-265, Stanford Linear Accelerator Center, Stanford, CA, 1985.

${ }^{6}$ I. Kawrakow, "Accurate condensed history Monte Carlo simulation of electron transport. I. EGSnrc, the new EGS4 version," Med. Phys. 27, 485-498 (2000).

${ }^{7}$ M. J. Berger and S. M. Seltzer, ETRAN, Monte Carlo code system for electron and photon transport through extended media. RISC computer code package CCC-107, Oak Ridge National Laboratory, Oak Ridge, TN, 1973.

${ }^{8}$ A. Fasso, A. Ferrari, and P. R. Sala, Electron-photon transport in FLUKA: Status. in Proceedings of the Monte Carlo 2000 Conference, Lisbon, Portugal, edited by A. Kling, F. J. C. Barao, M. Nakagawa, L. Tavora, and P. Vaz (Springer-Verlag, Berlin, 2000), pp. 159-164.

${ }^{9}$ M. Goosens, S. Giani, and S. Ravndal, GEANT: detector description and simulation tool. Technical Report CERN Program Library, long writeup W5013 CERN, Geneva, Switzerland, 1993.

${ }^{10}$ J. A. Halblieb and T. A. Mehlhorn, ITS: The Integrated TIGER Series of coupled electron/photon Monte Carlo transport codes, Sandia National Laboratory report, SAND 84-0573, 1984.

${ }^{11}$ MCNP_-A general Monte Carlo N-Particle transport code, edited by J. F. Briesmeister Los Alamos National Laboratory Report LA-12625-M, 1993.

${ }^{12}$ F. Salvat, J. M. Fernández-Varea, J. Baró, and J. Sempau, "PENELOPE, an algorithm and computer code for Monte Carlo simulation of electronphoton showers," in Ciemat (Centro de Investigaciones Energéticas, Medioambientales y Tecnológicas), Report No. 799, 1992.

${ }^{13}$ C. M. Ma, J. S. Li, T. Pawlicki, S. B. Jiang, and J. Deng, "MCDOSE-a Monte Carlo dose calculation tool for radiation therapy treatment planning," in Proceedings of the XIII International Conference on the Use of Computer in Radiation Therapy (ICCR), edited by W. Schlegel and T. Bortfeld (Springer-Verlag, Heidelberg), pp. 123-125.

${ }^{14}$ L. Wang, C. S. Chui, and M. Lovelock, "A patient-specific Monte Carlo dose-calculation method for photon beams," Med. Phys. 25, 867-878 (1998)

${ }^{15}$ J. Sempau, S. J. Wilderman, and A. F. Bielajew, "DPM, a fast, accurate Monte Carlo code optimized for photon and electron radiotherapy treatment planning dose calculations," Phys. Med. Biol. 45, 2263-2291 (2000).

${ }^{16}$ A. E. Schach von Wittetnau, L. J. Cox, P. M. Bergstrom, W. P. Chandler, C. L. Hartmann-Siantar, and R. Mohan, "Correlated histogram representation of Monte Carlo derived medical accelerator photon-output phase space,” Med. Phys. 26, 1196-1211 (1999).

${ }^{17}$ C. L. Hartmann Siantar, R. S. Walling, T. P. Daly, B. Faddegon, N. Albright, Paul Bergstrom, A. F. Bielajew, C. Chuang, D. Garrett, R. K. House, D. Knapp, D. J. Wieczorek, and L. J. Verhey, "Description and dosimetric verification of the PEREGRINE Monte Carlo dose calculation system for photon beams incident on a water phantom," Med. Phys. 28, 1322-1337 (2001).

18 "VMC ++ , electron and photon Monte Carlo calculations optimized for Radiation Treatment Planning" in Advanced Monte Carlo for Radiation Physics, Particle Transport Simulation and Applications: Proceedings of the Monte Carlo 2000 Meeting, Lisbon, edited by A. Kling, F. Barao, M. Nakagawa, L. Tavora, and P. Vaz (Springer, Berlin, 2001), pp. 229-236.

${ }^{19}$ M. Fippel, "Fast Monte Carlo dose calculation for photon beams based on the VMC electron algorithm," Med. Phys. 26, 1466-1475 (1999).

${ }^{20}$ H. Neuenschwander, T. R. Mackie, and P. J. Reckwerdt, "MMC-a highperformance Monte Carlo code for electron beam treatment planning," Phys. Med. Biol. 40, 543-574 (1995).

${ }^{21}$ P. J. Keall and P. W. Hoban, "Super-Monte Carlo: A 3D electron beam dose calculation algorithm," Med. Phys. 23, 2023-2034 (1996).

${ }^{22}$ D. W. O. Roger, B. A. Faddegon, G. X. Ding, C. M. Ma, J. Wei, and T. R. Mackie, "BEAM: A Monte Carlo code to simulate radiotherapy treatment units," Med. Phys. 22, 503-524 (1995).

${ }^{23}$ J. V. Siebers, P. J. Keall, B. Libby, and R. Mohan, "Comparison of EGS4 and MCNP4b Monte Carlo codes for generation of photon phase space distributions for a Varian 2100C," Phys. Med. Biol. 44, 3009-3026 (1999).

${ }^{24}$ C. M. Ma, "Characterization of computer simulated radiotherapy beams for Monte Carlo treatment planning," Radiation Phys. Chemistry 53, 329-344 (1998).

${ }^{25}$ A. E. Schach von Wittenau, P. M. Bergstrom, Jr., and L. J. Cox, "Patientdependent beam-modifier physics in Monte Carlo photon dose calculations," Med. Phys. 27, 935-947 (2000).

${ }^{26}$ E. R. Woodcock et al., in Argonne National Laboratory Report, ANL7050, 557, 1965.

${ }^{27}$ I. Lux and L. Koblinger, Monte Carlo Particle Transport Methods: Neutron and Photon Calculations (CRC, Boca Raton, 1991).

${ }^{28}$ J. J. DeMarco, T. D. Solberg, I. Chetty, and J. B. Smathers, "Efficient sampling algorithms for Monte Carlo based treatment planning," Radiat. Phys. Chem. 53, 229-234 (1998).

${ }^{29}$ I. Kawrakow and M. Fippel, "Investigation of variance reduction techniques for Monte Carlo photon dose calculation using XVMC," Phys. Med. Biol. 45, 2163-2184 (2000).

${ }^{30} \mathrm{M}$. Berger, "Monte Carlo Calculation of the penetration and diffusion of fast charged particles," Methods Comput. Phys. 135-215 (1963).

31 "Handbook on Photonuclear Data for Applications, Cross Sections and Spectra," IAEA-TECDOC—Draft No. 3, March 2000.

${ }^{32}$ J. A. Rawlinson, M. K. Islam, and D. M. Galbraith, "Dose to radiation therapists from activation at high-energy accelerators used for conventional and intensity-modulated radiation therapy," Med. Phys. 29, 598608 (2002).

${ }^{33}$ D. Followill, P. Geis, and A. Boyer, "Estimates of whole-body dose equivalent produced by beam intensity modulated conformal therapy," Int. J. Radiat. Oncol., Biol., Phys. 38, 667-672 (1997).

${ }^{34} \mathrm{P}$. McGinley and K. E. Huffman, "Photon and neutron dose equivalent in the maze of a high-energy medical accelerator facility," Radiat. Prot. Manage. 17, 43-46 (2000)

${ }^{35}$ ICRU: Report No. 44, "Tissue Substitutes in Radiation Dosimetry and Measurement," Washington, D.C., International Commission on Radiation Units and Measurements, 1989.

${ }^{36}$ W. Schneider, T. Bortfeld, and W. Schlegel, "Correlation between CT number and tissue parameters needed for Monte Carlo simulations of clinical dose distributions," Phys. Med. Biol. 45, 459-478 (2000).

${ }^{37}$ C. M. Ma, B. A. Faddegon, D. W. O. Rogers, and T. R. Mackie, "Accurate characterization of Monte Carlo calculated electron beams for radiotherapy," Med. Phys. 24, 401-416 (1997).

${ }^{38}$ B. Faddegon, J. Balogh, R. Mackenzie, and D. Scora, "Clinical considerations of Monte Carlo for electron radiotherapy treatment planning," Radiat. Phys. Chem. 53, 217-227 (1998).

${ }^{39}$ I. Chetty, J. J. DeMarco, and T. D. Solberg, “A virtual source model for Monte Carlo modeling of arbitrary intensity distributions," Med. Phys. 27, 166-172 (2000)

${ }^{40}$ J. Deng, S. B. Jiang, J. S. Li, T. Pawlicki, and C. M. Ma, "Photon beam characterization and modeling for Monte Carlo treatment planning," Phys. Med. Biol. 45, 411-427 (2000).

${ }^{41}$ M. K. Fix, M. Stampanoni, P. Manser, R. J. Born, R. Mini, and P. Ruegsegger, "A multiple source model for $6 \mathrm{MV}$ photon beam dose calculations," Phys. Med. Biol. 46, 1407-1428 (2001).

${ }^{42}$ S. B. Jiang, J. Deng, J. S. Li, P. Pawlicki, A. L. Boyer, and C. M. Ma, "Modeling and Commissioning of Clinical Photon Beams for Monte Carlo Treatment Planning," in Proceedings of the XIII International Conference on the Use of Computer in Radiation Therapy (ICCR), edited by W. Schlegel and T. Bortfeld (Springer-Verlag, Heidelberg, 2000), pp. 434-436.

${ }^{43}$ S. B. Jiang, A. L. Boyer, and C. M. Ma, "Modeling the extrafocal radiation and monitor chamber backscatter for photon beam dose calculation," Med. Phys. 28, 55-66 (2001).

${ }^{44}$ J. Deng, S. B. Jiang, P. Pawlicki, J. S. Li, and C. M. Ma, "Electron beam commissioning for Monte Carlo dose calculation," in Proceedings of the XIII International Conference on the Use of Computer in Radiation Therapy (ICCR), edited by W. Schlegel and T. Bortfeld (Springer-Verlag, Heidelberg, 2000), pp. 431-433. 
${ }^{45}$ J. J. Fischer and J. E. Moulder, "The steepness of the dose-response curve in radiation therapy," Radiology 117, 179-184 (1975).

${ }^{46}$ L. J. Shukovsky, "Dose, time, volume relationships in squamous cell carcinoma of the supraglottic larynx," Am. J. Roentgenol. 108, 27-29 (1970).

${ }^{47}$ ICRU: Report No. 24, "Determination of Absorbed Dose in a Patient Irradiated by Beams of X or Gamma Rays in Radiotherapy Procedures," Washington, D.C., International Commission on Radiation Units and Measurements, 1976

${ }^{48}$ R. Loevinger and T. P. Loftus, "Uncertainty in the delivery of absorbed dose," in Ionizing Radiation Metrology, International Course, edited by E. Casnati (Varenna, Italy, 1974), pp. 459-473 (Bologna, Editrice Compositore, 1977).

${ }^{49}$ M. Langer and P. Kijewski, "Computer controlled radiation therapy for non-small cell lung cancer: Sensitivity of tumor dose gains to pulmonary dose-volume restrictions," Int. J. Radiat. Oncol., Biol., Phys. 22, 325332 (1992)

${ }^{50}$ M. Langer, P. Kijewski, R. Brown, and C. Ha, "The effect on minimum tumor dose of restricting target dose inhomogeneity in optimized 3-dimensional treatment of lung cancer," Radiother. Oncol. 21, 245-256 (1991).

${ }^{51}$ C. M. Ma, E. Mok, A. Kapur, T. Pawlicki, D. Findley, S. Brain, K. Forster, and A. L. Boyer, "Clinical implementation of a Monte Carlo treatment planning system," Med. Phys. 26, 2133-2143 (1999).

${ }^{52}$ P. J. Keall, J. V. Siebers, M. Arnfield, J. O. Kim, and R. Mohan, "2001 Monte Carlo dose calculations for dynamic IMRT treatments," Phys. Med. Biol. 46, 929-934 (2001).

${ }^{53}$ J. V. Siebers, P. J. Keall, J. O. Kim, and R. Mohan, "A method for photon beam Monte Carlo multileaf collimator particle transport," Phys. Med. Biol. 47(17), 3225-3249 (2002)

${ }^{54}$ T. D. Solberg, J. J. DeMarco, J. B. Smathers, F. E. Holly, A. A. F. DeSalles, "Monte Carlo Treatment Planning for Stereotactic Radiosurger," Radiother. Oncol. 49, 73-84 (1998).

${ }^{55}$ G. Ding, "Dose discrepencies between Monte Carlo calculations and measurements in the buildup region for a high energy photon beam," Med. Phys. 29, 2459-2463 (2002).

${ }^{56}$ B. Fraass, K. Doppke, M. Hunt, G. Kutcher, G. Starkschall, R. Stern, and J. Van Dyke, "American Association of Physicists in Medicine Radiation Therapy Committee Task Group 53: Quality assurance for clinical radiotherapy treatment planning," Med. Phys. 25, 1773-1829 (1998).

${ }^{57}$ R. Rice, B. Mijnheer, and L. Chin, "Benchmark measurements for lung dose corrections for x-ray beams," Int. J. Radiat. Oncol., Biol., Phys. 15, 399-409 (1988).
${ }^{58}$ MCNPX User's Manual, Version 2.4.0, LA-CP-02-408 (Los Alamos National Laboratory, Los Alamos, NM, 2003).

${ }^{59}$ I. J. Chetty, J. M. Moran, T. Nurushev, D. L. McShan, B. A. Fraass, S. J. Wilderman, and A. F. Bielajew, "Experimental validation of the dose planning method (DPM) Monte Carlo code using minimally scattered electron beams in heterogeneous media," Phys. Med. Biol. 47, 18371851 (2002).

${ }^{60}$ I. J. Chetty, P. M. Charland, N. Tyagi, D. L. McShan, A. F. Bielajew, and B. A. Fraass, "Photon beam relative dose validation of the DPM Monte Carlo code in lung-equivalent media," Med. Phys. 30, 563-573 (2003).

${ }^{61}$ M. Fippel, I. Kawrakow, and K. Friedrich, "Electron beam dose calculations with the VMC algorithm and the verification data of the NCI working group," Phys. Med. Biol. 42, 501-520 (1997).

${ }^{62}$ J. S. Li, T. Pawlicki, J. Deng, S. B. Jiang, E. Mok, and C. M. Ma, "Validation of a Monte Carlo dose calculation tool radiotherapy treatment planning," Phys. Med. Biol. 45, 2969-2985 (2000).

${ }^{63}$ J. J. DeMarco, T. D. Solberg, J. B. Smathers, "A CT-based Monte Carlo simulation tool for dosimetry planning and analysis," Med. Phys. 25, $1-11$ (1998).

${ }^{64}$ J. V. Siebers, P. J. Keall, J. O. Kim, R. Mohan, "Performance benchmarks of the MCV Monte Carlo system," in Proceedings of the XIIIth International Conference on the Use of Computers in Radiation Therapy, edited by W. Schlegel and T. Bortfeld (Springer-Verlag, Berlin, Germany, 2000), pp. 129-131.

${ }^{65}$ M. R. Arnfield, C. L. Hartmann-Siantar, J. Siebers, P. Garmon, L. Cox, and R. Mohan, "The impact of electron transport on the accuracy of computed dose," Med. Phys. 27, 1266-1274 (2000).

${ }^{66} \mathrm{~L}$. Wang, M. Lovelock, and C. S. Chui, "Experimental verification of a CT-based Monte Carlo dose-calculation method in heterogeneous phantoms," Med. Phys. 26, 2626-2634 (1999).

${ }^{67}$ C. M. Ma, P. Reckwerdt, M. Holmes, D. W. O. Rogers, and B. Geiser, DOSXYZ Users Manual. NRC Report PIRS 509b, 1998.

${ }^{68}$ M. K. Fix, H. Keller, E. J. Born, and P. Ruegsegger, "Simple beam models for Monte Carlo photon beam dose calculations in radiotherapy," Med. Phys. 27, 2739-2747 (2000).

${ }^{69}$ J. M. Michalski et al., "Preliminary report of toxicity following: 3D radiation therapy for prostate cancer on 3DOG/RTOG 9406," Int. J. Radiat. Oncol., Biol., Phys. 46, 391-402 (2000).

${ }^{70}$ J. A. Purdy, W. B. Harm, J. M. Michalski, and W. R. Bosch, "Initial experience with quality assurance of multi-Institutional 3D radiotherapy clinical trials," Strahlenther. Onkol. 174, 40-42 (1998). 